\title{
Endoscopic diverticulotomy for Killian-Jamieson diverticulum: mid-term outcome and description of an ultra-short tunnel technique $\nabla$
}

\section{(๑) $\odot \ominus$}

\author{
Authors \\ Institutions \\ 1 Department of Gastroenterology, Hepatology, and \\ Nutrition, NYU-Winthrop Hospital, Mineola, New York, \\ United States \\ 2 Department of Medicine, Mount Sinai St Luke's-West \\ Hospital Center, New York, New York, United States \\ 3 Department of Medicine, division of Gastroenterology, \\ University of Missouri Health Care, University of \\ Missouri, Columbia, Missouri, United States \\ 4 Division of Gastroenterology and Hepatology, Elmhurst \\ Hospital- Mount Sinai, Elmhurst, New York, United \\ States
}

Rani J. Modayil ${ }^{1}$, Xiaocen Zhang ${ }^{2}$, Mohammad Ali ${ }^{1}$, Kanak Das ${ }^{1,3}$, Krishna Gurram4, Stavros N. Stavropoulos ${ }^{1}$

\section{ABSTRACT} therapy. submitted 24.3.2020

accepted after revision 24.6.2021

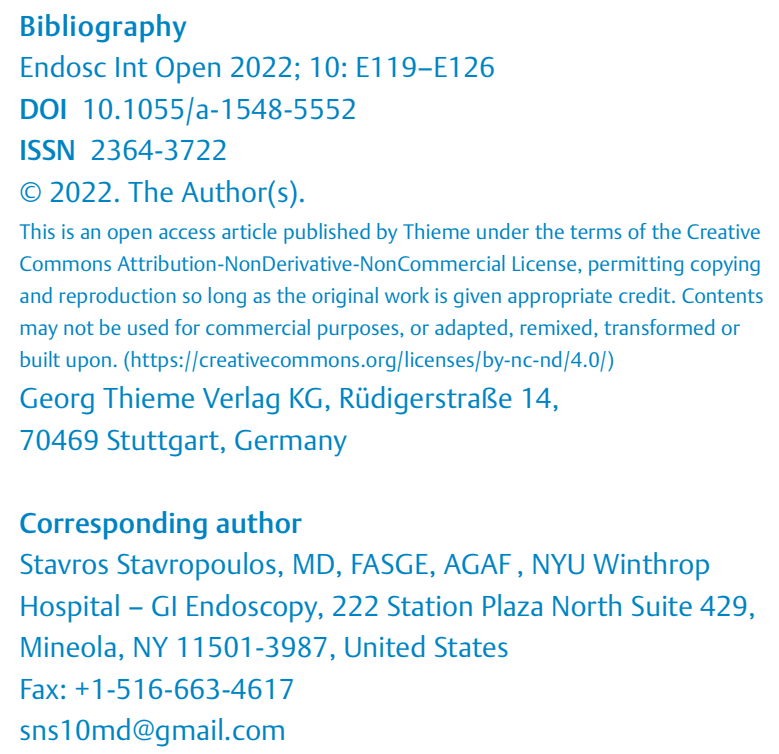

This is an open access article published by Thieme under the terms of the Creative Commons Attribution-NonDerivative-NonCommercial License, permitting copying and reproduction so long as the original work is given appropriate credit. Contents may not be used for commercial purposes, or adapted, remixed, transformed or built upon. (https://creativecommons.org/licenses/by-nc-nd/4.0/)

Georg Thieme Verlag KG, Rüdigerstraße 14,

70469 Stuttgart, Germany

Corresponding author

Stavros Stavropoulos, MD, FASGE, AGAF, NYU Winthrop

Hospital - GI Endoscopy, 222 Station Plaza North Suite 429,

Mineola, NY 11501-3987, United States

Fax: +1-516-663-4617

sns10md@gmail.com

Background and study aims Killian-Jamieson Diverticulum (KJD) is a rarer and more recently described upper pharyngeal diverticulum than Zenker's diverticulum (ZD). $\mathrm{KJD}$ is more difficult to manage than ZD because it tends to extend lower into the upper mediastinum and the diverticulum neck is in close proximity to the recurrent laryngeal nerve. There is limited literature on KJD management and transcervical surgical diverticulectomy is the mainstay of

Patients and methods Here we describe two methods of endoscopic diverticulotomy to treat KJD - direct and tunneling diverticulotomy (with hypopharyngeal tunnel or ultra-short tunnel - the latter being our preferred technique). Results This was a retrospective study including 13 consecutive patients between March 2015 and April 2018. Three patients received direct and 10 received tunneling diverticulotomy ( 7 with the hypopharyngeal tunnel and 3 with the ultra-short tunnel). All procedures were completed in 16 to 52 minutes. There was no incidence of bleeding, mediastinitis, or sign of recurrent laryngeal nerve injury. At follow up of 9 to 79 months (median 33), the clinical success rate was $92 \%$ (12/13); 11 patients had complete symptom resolution (post-operative symptom score $=0$ ) and one patient had near-complete symptom resolution (occasional residual dysphagia). One patient receiving direct myotomy had limited symptom relief (frequent residual dysphagia and occasional residual regurgitation), possibly related to incomplete myotomy.

Conclusions Endoscopic tunneling diverticulotomy is a feasible, safe, and effective method to treat KJD.

\section{Introduction}

Killian-Jamieson diverticulum (KJD) is a rare pharyngoesophageal diverticulum. First described in 1983 [1], it is an anterolateral outpouching of mucosa at the Killian-Jamieson triangle, an anatomical weakness inferior to the transverse portion of the cricopharyngeal muscle and lateral to the longitudinal esophageal muscle. Compared to the more common Zenker's diverticulum (ZD), which is a posterolateral outpouching through the Killian dehiscence (an area of weakness between the cricopharyngeus distally and thyropharyngeal and inferior constrictor muscles proximally), KJD is located more distally and tends to be antero-lateral rather than posterior [2] and the age of presentation tends to be younger [3,4] (> Fig.1, 


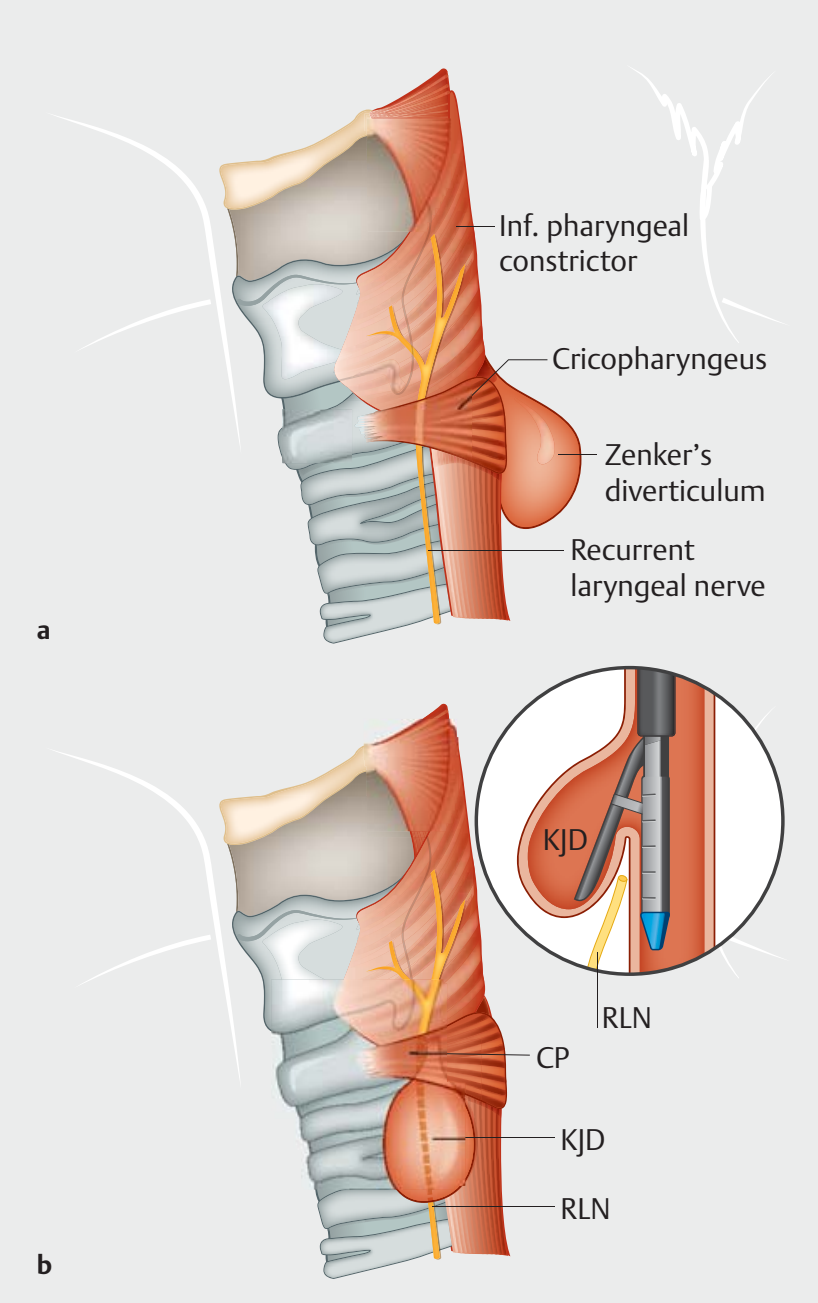

- Fig. 1 a and $\mathbf{b}$ ZD and KJD. ZD originates above the cricopharyngeal muscle and tends to be posterior. KJD originates below the cricopharyngeal muscle and tends to be lateral or anterior. The neck of KJD is in close proximity to the recurrent laryngeal nerve, making stapler trans-oral diverticulotomy risky.
- Table 1). Due to its rarity (having a reported incidence of onequarter of that of ZD [2]), KJD is often misdiagnosed as ZD on radiographs. However, the two entities are normally readily discernable on endoscopic examination because KJD is located below the cricopharyngeal bar and ZD above the bar. Differentiating the two entities is important for two reasons: 1 . In KJD the recurrent laryngeal nerve passes in very close proximity to the neck of the diverticulum and may be injured particularly if a transoral rigid stapled diverticulotomy is attempted (> Fig. 1); and 2. KJD tends to extend into the upper mediastinum; therefore, a leak may result in mediastinitis, a more morbid adverse event than would occur from a leak after Zenker's diverticulotomy, which tends to be limited to the neck [5]. These are important considerations when choosing a treatment plan. ZD and KJD have similar clinical presentations: dysphagia, globus sensation in the throat, halitosis, regurgitation, cough and aspiration. The hypothesized pathogenesis for the two diseases is also similar: a functional outflow obstruction caused by dysfunction of the cricopharyngeal or upper esophageal muscles respectively [6]. For ZD, removal of the diverticulum itself used to be part of the treatment paradigm but is now considered unnecessary with various forms of cricopharyngeal myotomy alone, reportedly achieving good symptom relief and with flexible endoscopic diverticulotomy having emerged as the treatment of choice [7-9]. Due to its rarity and recent description, there is limited published literature on the treatment of $\mathrm{KJD}$ (60 or so publications in the English literature, primarily single case reports with follow up $\leq 3$ months) [4]. The mainstay treatment for KJD is transcervical diverticulotomy, given concern about recurrent laryngeal nerve injury and mediastinitis if per-oral diverticulum myotomy is to be used. Herein we present a series of $13 \mathrm{KJD}$ patients treated with endoscopic diverticulotomy, the largest report to date on KJD treatment of any kind.

\section{Patients and methods}

From a prospectively maintained database of endoscopic myotomy procedures, we identified all diverticulotomies for KJD. We performed the first such procedure in February 2014. The study was approved on April 26, 2018 by NYU Winthrop IRB

- Table 1 Differences between Killian Jamieson and Zenker's diverticulum. Based on a recent literature review [4].

\begin{tabular}{|l|l|l|}
\hline & Killian Jamieson (KJ) diverticulum & Zenker diverticulum (ZD) \\
\hline Location & $\begin{array}{l}\text { Lateral wall of pharyngoesophageal junction in space below cri- } \\
\text { copharyngeus muscle and lateral to longitudinal muscular fiber } \\
\text { of esophagus }\end{array}$ & $\begin{array}{l}\text { Posterior wall of pharnygoesophageal junction below su- } \\
\text { perior pharyngeal constrictor muscle and above cricophar- } \\
\text { yngeus muscle }\end{array}$ \\
\hline Prevalence & Rare & More Common \\
\hline Laterality & Left & Left \\
\hline Gender & Female & Male \\
\hline Age average & 58 years & 70 years \\
\hline Size & Small & Medium to Large \\
\hline Symptoms & $\begin{array}{l}\text { Dysphagia, regurgitation, globus sensation } \\
\text { At least one-third have no aerodigestive symptoms }\end{array}$ & $\begin{array}{l}\text { Dysphagia, cervical borborygmus, weight loss, hoarseness, } \\
\text { cough, aspiration pneumonia }\end{array}$ \\
\hline
\end{tabular}



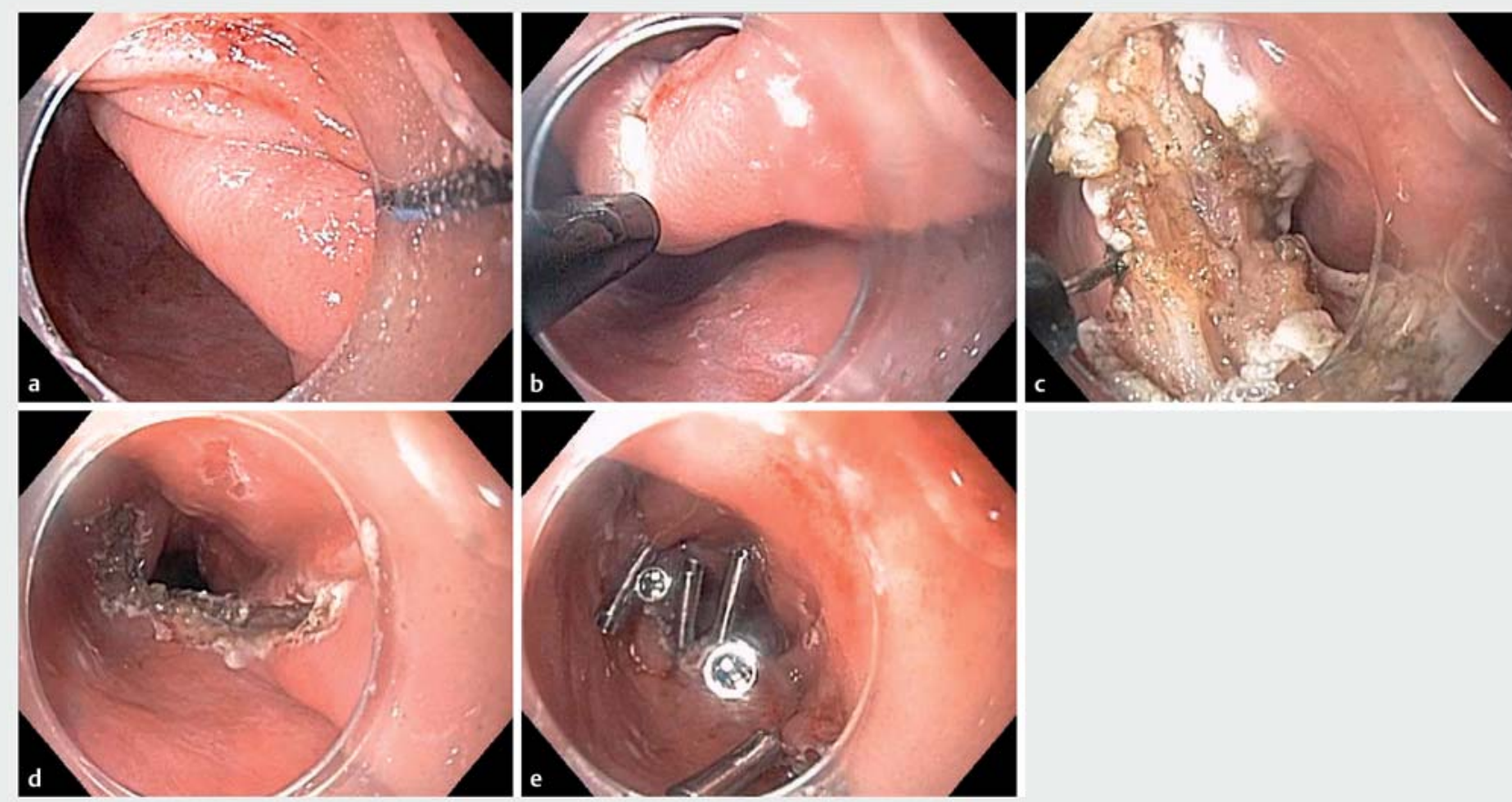

Fig. 2 a, b, c, d, e direct endoscopic diverticulotomy using the triangular tip knife. The mucosa is approximated with clips after completion of the myotomy.

(\#03125733) to include data in a research registry including those previously and all subsequently collected. Patients with a diagnosis of KJD based on clinical symptoms and esophagram and endoscopic findings were included. Patients with coagulopathy or for whom diverticulotomy would have offered limited benefit because of severe comorbidities, limited life expectancy, or mild symptoms were excluded. A total of 13 procedures were identified in the study period from February 2014 to October 2018. Patients were evaluated for the following symptoms: dysphagia for solids (D), regurgitation (R), unexplained cough or other respiratory symptoms suspicious for aspiration, or choking sensation while eating (C), and weight loss (W). Symptoms $\mathrm{D}, \mathrm{R}$ and $\mathrm{C}$ were scored according to their frequency of occurrence: absent $=0$, occasional $=1$, frequent $=2$ and daily (or food impaction for dysphagia) $=3$. Weight loss was recorded. Post-myotomy weight change was compared to the weight at the time of myotomy.

Two types of endoscopic diverticulotomy have been used to treat KJD in our center: direct diverticulotomy and tunneling diverticulotomy. Direct diverticulotomy is an established method to treat ZD [10-14] and has been reported in KJD patients in single case reports [15-18]. It was used in the beginning of our study period. Our preferred method changed to tunneling myotomy because of its perceived better efficacy and safety profile.

In endoscopic direct diverticulotomy, all the layers of the diverticular septum (mucosa, submucosa, and upper esophageal muscle) are dissected to approximately 5 to $10 \mathrm{~mm}$ proximal to the level of the fundus of the diverticulum. After completion of the septotomy, the mucosal edges of the cut are approximated with clips to minimize the risk of leak ( $\vee$ Fig. 2, $\triangleright$ Video 1, $>$ Table 2).

In endoscopic tunneling diverticulotomy, myotomy is performed using a technique similar to that of peroral endoscopic myotomy (POEM). There are two variations. Our initial case was performed by initiating the tunnel in the hypopharynx proximal to the septum of the diverticulum ( $\vee$ Fig. 3, $\triangleright$ Video 2 , $\triangleright$ Table 2). This technique has also been reported for ZD [19-23]. However, we subsequently modified the technique so as to initiate the tunnel at the septum of the diverticulum ( $\mathbf{F i g . 4 , ~} \vee$ Video 2, > Table 2) because it was evident that starting the tunnel at the diverticular septum rather than more proximally in the hypopharynx allows immediate ready access to the muscle of the

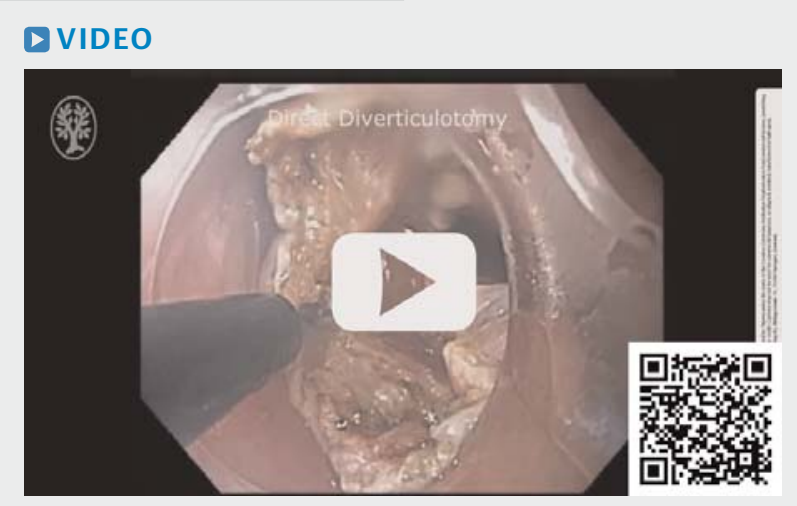

- Video 1 Direct endoscopic diverticulotomy technique. 
- Table2 Three techniques for endoscopic diverticulotomy for hypopharyngeal diverticulum.

\begin{tabular}{|c|c|c|c|}
\hline & Direct septotomy & Tunnel starting in hypopharynx & Tunnel starting at septum \\
\hline Technique & $\begin{array}{l}\text { Performing septotomy through } \\
\text { mucosa, submucosa and muscle } \\
\text { and stopping } 10 \mathrm{~mm} \text { proximal to } \\
\text { fundus of diverticulum to avoid } \\
\text { mediastinal entry }\end{array}$ & $\begin{array}{l}2 \mathrm{~cm} \text { submucosal tunnel prior to } \\
\text { reaching the muscle and initiat- } \\
\text { ing the myotomy. A true submu- } \\
\text { cosal tunnel is formed upstream } \\
\text { of the myotomy site. }\end{array}$ & $\begin{array}{l}\text { Entry at septum and the tunnel is formed as the } \\
\text { myotomy is initiated and as the mytomy is extended } \\
\text { the tunnel is extended. The tunnel is formed by the } \\
\text { myotomy since the beginning of the tunnel is at the } \\
\text { apex of the septum. }\end{array}$ \\
\hline Knife & $\begin{array}{l}1^{\text {st }} \text { case triangle tip knife and I } \\
\text { type } \\
\text { hybrid knife } \\
2^{\text {nd }} \text { case triangle tip knife and dual } \\
\text { knife } \\
3^{\text {rd }} \text { case triangle tip knife }\end{array}$ & $\begin{array}{l}4^{\text {th }} \text { case Hook knife } \\
5^{\text {th }} \text { case I type hybrid knife } \\
6^{\text {th }} \text { case I type hybrid knife and } \\
\text { hook knife } \\
7^{\text {th }} \text { case I type hybrid knife } \\
8^{\text {th }} \text { case I type hybrid knife } \\
9^{\text {th }} \text { case Hook knife } \\
10^{\text {th }} \text { case Hook knife }\end{array}$ & $\begin{array}{l}11^{\text {th }} \text { case Hook knife } \\
12^{\text {th }} \text { case Hook knife } \\
13^{\text {th }} \text { case I type hybrid knife }\end{array}$ \\
\hline Closure $^{1}$ & $\begin{array}{l}\text { First two cases no closure per- } \\
\text { formed } \\
\text { Third case } 5 \text { endoclips and tisseel } \\
\text { fibrin sealant }\end{array}$ & $\begin{array}{l}4^{\text {th }} \text { case- } 5 \text { endoclips } \\
5^{\text {th }} \text { case } 5 \text { endoclips } \\
6^{\text {th }} \text { case } 3 \text { endoclips } \\
7^{\text {th }} \text { case } 6 \text { endoclips and tisseel } \\
\text { fibrin sealant } \\
8^{\text {th }} \text { case } 7 \text { endoclips } \\
9^{\text {th }} \text { case } 4 \text { endoclips } \\
10^{\text {th }} \text { case } 6 \text { endoclips }\end{array}$ & $\begin{array}{l}11^{\text {th }} \text { case } 4 \text { endoclips } \\
12^{\text {th }} \text { case } 4 \text { endoclips } \\
13^{\text {th }} \text { case } 6 \text { endoclips }\end{array}$ \\
\hline Advantages & $\begin{array}{l}\text { Technically simple } \\
\text { Fast }\end{array}$ & $\begin{array}{l}\text { Secure closure } \\
\text { Allows for longer myotomy }\end{array}$ & $\begin{array}{l}\text { Secure closure } \\
\text { Allows for long myotomy } \\
\text { Easier closure than in the hypopharyngeal tunnel }\end{array}$ \\
\hline $\begin{array}{l}\text { Disadvanta- } \\
\text { ges }\end{array}$ & $\begin{array}{l}\text { Less secure closure, higher risk of } \\
\text { leak } \\
\text { Short myotomy, higher risk of re- } \\
\text { currence }\end{array}$ & $\begin{array}{l}\text { More challenging tunnel closure } \\
\text { than the ultra-short tunnel }\end{array}$ & $\begin{array}{l}\text { Secure closure } \\
\text { Allows for long myotomy } \\
\text { Easier closure than in the hypopharyngeal tunnel }\end{array}$ \\
\hline
\end{tabular}

septum and permits easier closure of the tunnel orifice than is possible in the hypopharynx, where there is less space to maneuver the endoscope, access to the tunnel orifice is more tangential, and tissue is less mobile. Another difference from the ZD technique presented by Li et al is that we perform the myotomy and tunneling concurrently rather than sequentially.

In direct diverticulotomy, the Triangle Tip Knife (Olympus America, KD-640 L) was preferably used; in tunneling diverticulotomy, the HookKnife (Olympus America, KD-620LR) or HybridKnife (ERBE USA, 20150-261) was preferably used. During direct diverticulotomy, dissection was performed with spray coagulation (effect 2, 45 watts), and Endocut I (effect 1, duration 3, interval 1) was used for fibrotic areas with poor conductance. During tunneling diverticulotomy, tunneling was performed with Endocut I (effect 1, duration 3, interval 1) and myotomy was performed with spray coagulation (effect 2, 45 watts). In both direct and tunneling diverticulotomy, a Coagrasper (Olympus America, FD-410LR) with soft coagulation (effect 5,80 watts) was used as needed to achieve hemostasis. A transparent cap at the tip of the endoscope was used to facilitate visualization and endoscopic maneuvering. The 4-mm straight ESD cap (Olympus America, D-201) was initially used. This was changed to the tapered-tip ST hood (Fujifilm Japan, DH-28 R) when it became available because it allowed for easier access into the submucosal space. A diverticuloscope was not used as it was not deemed to offer any advantages over the distal transparent cap attachment in this clinical application.

All procedures were performed by a single operator with extensive experience of POEM and ESD. General anesthesia with endotracheal intubation was used and patients were monitored in the hospital postoperatively. An esophagram with gastrograffin was performed for all patients before a diet was resumed. Broad-spectrum antibiotics were used perioperatively. Patients were seen in clinic 1 month postoperatively and telephone follow-up was performed at 6 months and then yearly after.

\section{Results}

Thirteen KJD patients received endoscopic diverticulotomy between February 2014 and October 2018 in our center. There were seven men and six women. The median age was 71 years (range 50-94). Four patients were ASA class II and 9 were ASA class III. Duration of disease ranged from 6 months to 30 years (median 3 years). Based on preoperative Barium esophagram radiologic interpretation, the cephalocaudal length of the diverticulum ranged from 1.5 to $6 \mathrm{~cm}$ and four patients were reported to have an anterior diverticulum, four a right antero-lat- 

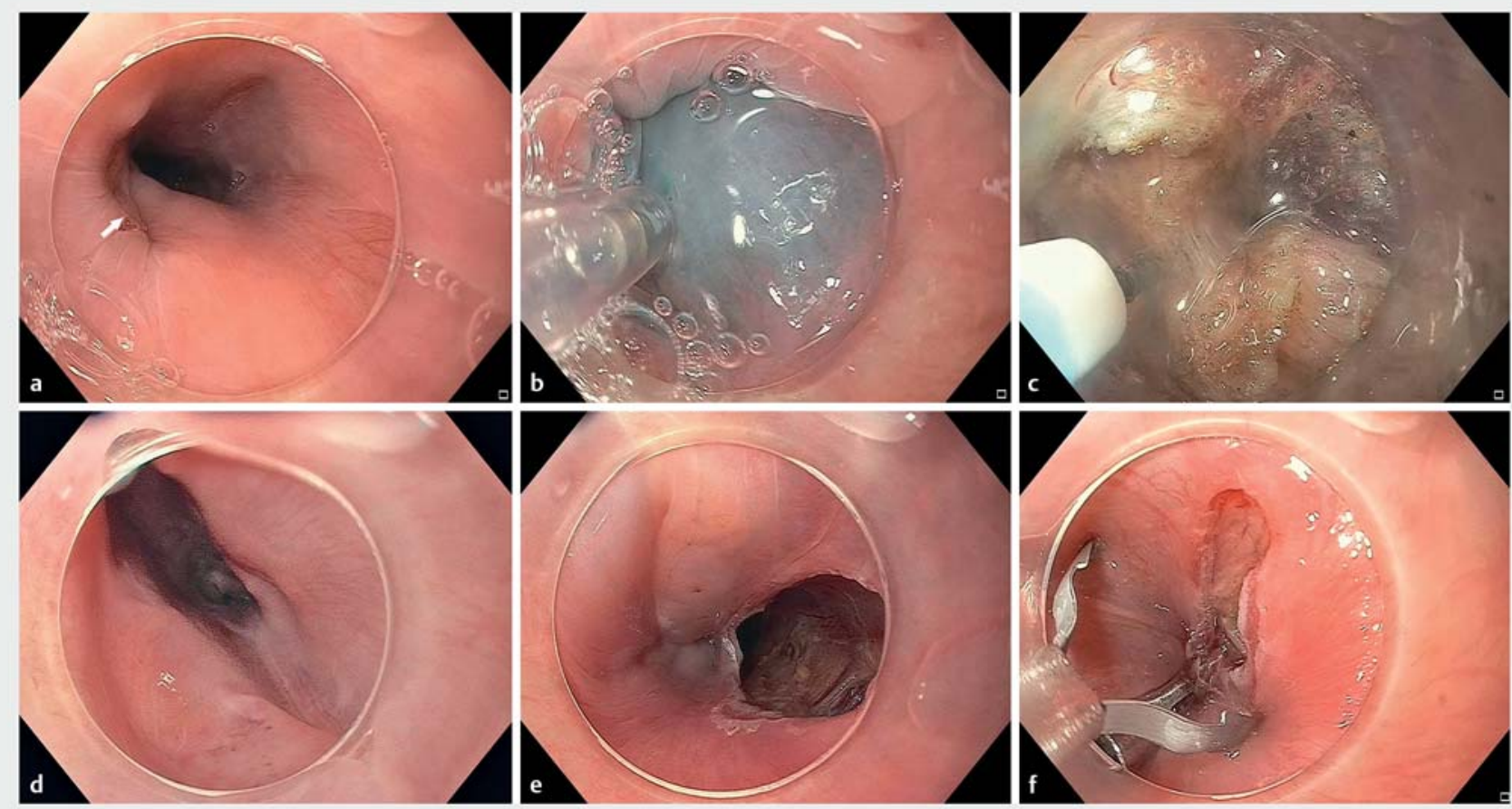

- Fig. 3 A case of tunneling diverticulotomy with hypopharyngeal tunnel. a An upper pharyngeal diverticulum is seen left lateral to the true lumen and inferior to the cricopharyngeal muscle. b Submucosal injection at the hypopharynx $2 \mathrm{~cm}$ above the proximal end of the diverticulum septum. The operating space is extremely limited in this location, leading to difficulties in anchoring and maneuvering the endoscope. c Myotomy is performed concurrently with submucosal injection to delineate the muscle septum. There is not separate step of tunneling, as compared to the standard technique of sequential tunneling and myotomy for peroral endoscopic myotomy (or the technique reported by Li et al for Zenker's diverticulum). d The diverticulum disappeared at the end of the myotomy. e Mucosal defect at the tunnel entrance. $\mathbf{f}$ Endoscopic clips were used to close the defect.

eral and three a left antero-lateral diverticulum. Preoperatively all patients reported frequent to daily dysphagia, nine patients reported occasional to frequent regurgitation, and six patients reported occasional to daily food-related cough or choking sensation or history of unexplained aspiration pneumonia.

The first three patients received direct myotomy and the following 10 tunneling myotomy. For the three direct diverticulotomies, procedure time ranged from 16 to 55 minutes (median 49). Patients resumed diet in 48 to 72 hours and were discharged in 2 to 8 days. For the 10 tunneling diverticulotomies, procedure time ranged from 19 to 52 minutes (median 27). Patients resumed diet in 24 to 120 hours and were discharged in 1 to 5 days.

There was no bleeding, mediastinitis, vocal cord paralysis or leak intraoperatively or postoperatively. One patient with a history of substance abuse was kept in the hospital for 8 days because of concern about non-compliance with diet after discharge and social issues. He resumed clear fluids 72 hours postoperatively and had no unexpected events. One patient resumed diet 120 hours postoperatively because of a pharyngeal tear caused by the echoendoscope for EUS done during the same session for an unrelated issue.

Patients were followed up for 9 to 79 months (median 33). Clinical success was achieved in 12 of 13 patients ( $92 \%$ ). Eleven patients had complete resolution of all symptoms and one pa- tient receiving tunneling myotomy reported a marked improvement in his dysphagia from daily dysphagia prior to treatment to occasional dysphagia after treatment, and complete resolution of the frequent regurgitation that he had prior to treatment ( $\triangleright$ Table 3 ). One patient receiving direct myotomy (the first patient in the series) was considered a failure as she reported persistent dysphagia despite improvement in regurgitation from frequent to occasional.

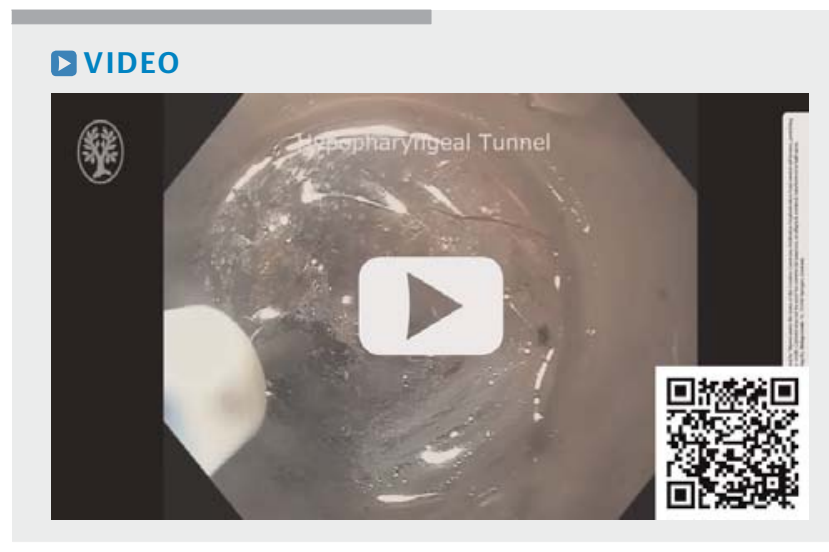

- Video 2 Tunneling Diverticulotomy. Two techniques demonstrated: Hypopharyngeal tunnel versus ultrashort septal tunnel. 

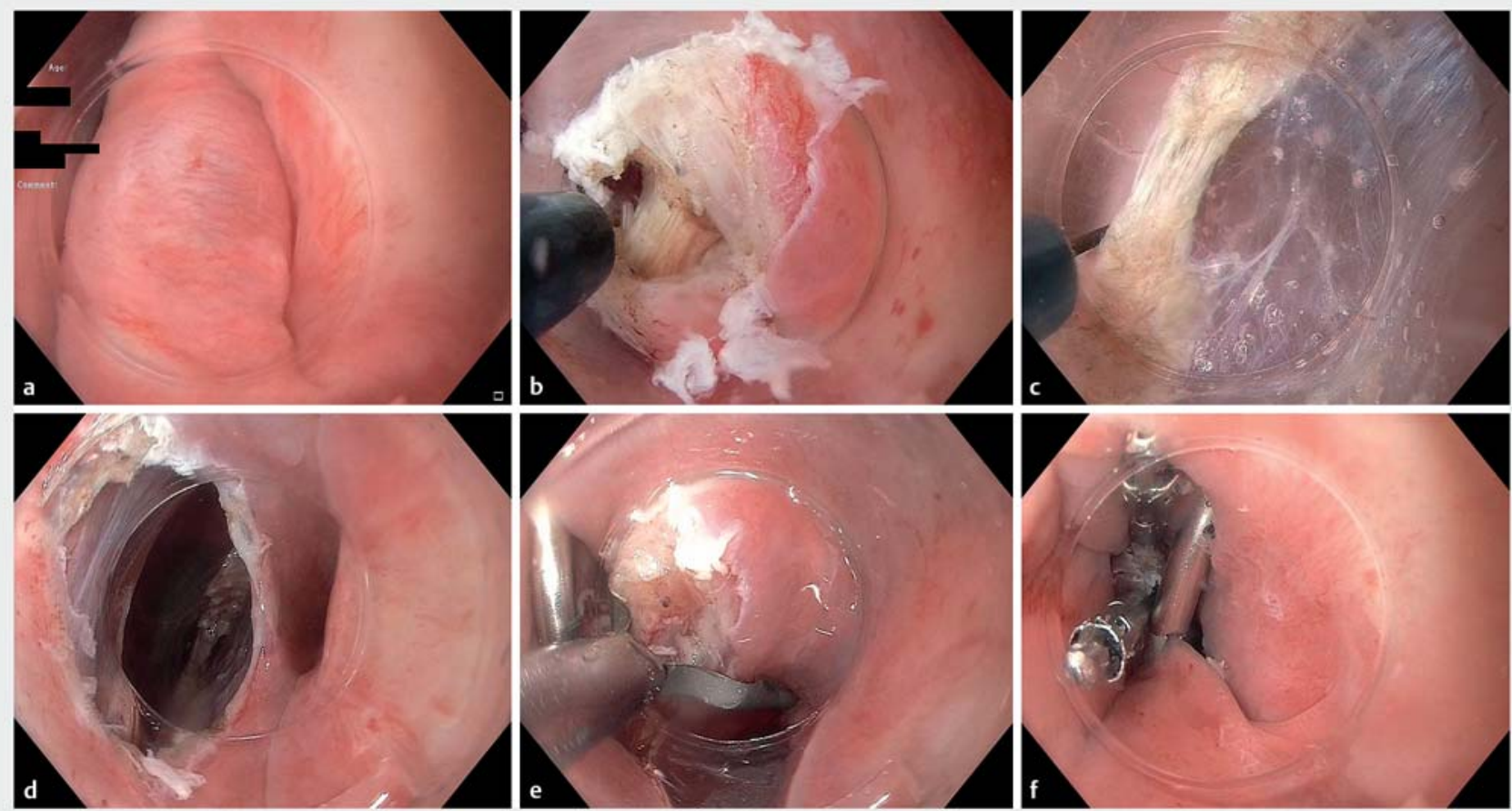

- Fig. 4 A case of tunneling diverticulotomy with ultra-short tunnel. a Submucosal injection at the apex of the septum. b Tunnel initiation is undertaken. After incision of the mucosa, injection is undertaken on both sides of the muscular septum to isolate and clearly define the muscle, which is then incised. Incision of the muscle is necessary to establish the tunnel proximally since the submucosal portion of the tunnel is only a few $\mathrm{mm}$ (equal to the thickness of the injected submucosa at the apex of the septum). c We perform simultaneous dissection of the muscle and submucosa on either side of the muscle extending the tunnel distally. $\mathbf{d}$ Completion of tunnel/myotomy. The end of the muscle incision at the tunnel terminus can be seen through the tunnel opening. e, $\mathbf{f}$ Tunnel closure with clips is facilitated by the en face location of the tunnel opening in this ultra-short tunnel technique which is our preferred technique.

\section{Discussion}

$\mathrm{KJD}$ is a rare and recently described type of cricopharyngeal diverticulum. Compared to ZD, KJD is less likely to be symptomatic (probably due to its lower location) [2] but more challenging to treat due to its extension in the mediastinum and proximity to the recurrent laryngeal nerve. Here we reported on endoscopic diverticulotomy using a submucosal tunneling myotomy method similar to that used in POEM. Compared to direct diverticulotomy, tunneling diverticulotomy allows easy and secure closure of the mucosal defect at the end of the procedure. This enables the operator to safely carry a full length myotomy through the entire septum of the diverticulum. This complete myotomy may improve symptom relief and durability [10] as compared to direct diverticulotomy, in which the septotomy/ myotomy is not usually carried through the full extent of the septum because of fear of perforation and leak. Furthermore, endoscopic myotomy is performed under high magnification, with the ability to precisely identify structures before cutting, and thus minimize the risk of accidental laryngeal nerve injury. Our method varies from the one reported by Li et al [19] and others [20-24] in ZD as we start tunneling at the septum rather than the hypopharynx, which facilitates closure of the mucosa defect and expedites the procedure. The myotomy and tunneling are done concurrently rather than sequentially because there is limited space to do a complete tunnel. This further expedites the procedure compared to the technique reported by Li et al. In our series of three direct and 10 tunneling diverticulotomies, all procedures were completed in 19 to 55 minutes. A similar ultra-short tunnel technique was recently reported by Repici et al for "short-septum" (septum<20 mm) ZD [25]. There were no adverse events related to the KJD diverticulotomy, specifically, no mediastinitis or sign of recurrent laryngeal nerve injury. After a follow-up of 9 to 79 months, 12 of 13 patients reported excellent long-term symptom relief. The only patient who had partial symptom relief was the first patient who had direct myotomy, possibly secondary to incomplete myotomy. In this small case series, there was no apparent difference between the two methods in terms of operation time, time with nothing by mouth or length of hospital stay. However, we do want to note that tunneling myotomy with the ultra-short tunnel is our preferred method because it allows complete myotomy to the bottom of the diverticulum without fear of mediastinal leak. We believe that such complete myotomy is the key to obtaining durable correction of the functional outflow obstruction caused by the muscular septum. This is the largest report to date on KJD treatment. 


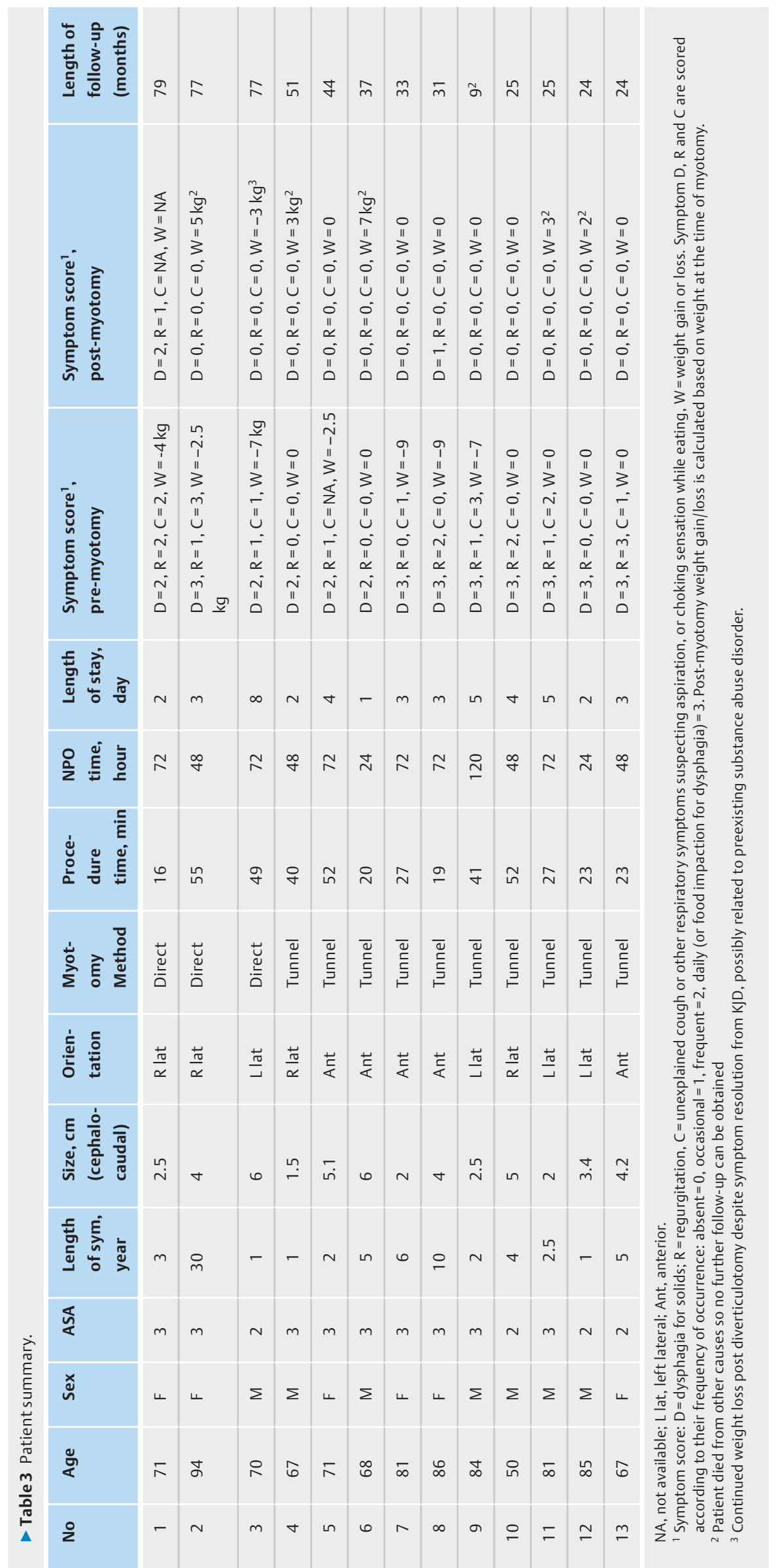




\section{Conclusions}

Endoscopic tunneling diverticulotomy is a feasible, safe, and effective method to treat KJD. The tunneling technique offers the theoretical benefit of more complete septotomy, and thus, long-term symptom resolution while minimizing the risk of accidental laryngeal nerve injury or leak. The "ultra-short tunnel" technique is a modification that is easier and faster than the more traditional "tunneling-followed-by-myotomy" technique employed in per-oral endoscopic myotomy. Because of the low incidence of the disease and scarcity of literature on it, a definitive benefit would be difficult to prove. However, in centers with relevant expertise, tunneling diverticulotomy can be an attractive alternative to surgical resection or direct endoscopic septotomy for patients with KJD.

\section{Competing interests}

Dr. Stavropoulos is a consultant for Boston Scientific and Olympus and receives honoraria from ERBE USA.

\section{References}

[1] Ekberg O, Nylander G. Lateral diverticula from the pharyngo-esophageal junction area. Radiology 1983; 146: 117-122

[2] Rubesin SE, Levine MS. Killian-Jamieson diverticula: radiographic findings in 16 patients. AJR Am J Roentgenol 2001; 177: 85-89

[3] Vogelsang A, Schumacher B, Neuhaus H. Therapy of Zenker's diverticulum. Dtsch Arztebl Int 2008; 105: 120

[4] Haddad N, Agarwal P, Levi JR et al. Presentation and management of killian jamieson diverticulum: a comprehensive literature review. Ann Otol Rhinol Laryngol 2020; 129: 394-400

[5] Undavia S, Anand SM, Jacobson AS. Killian-Jamieson diverticulum. The Laryngoscope 2013; 123: 414-417

[6] Peters ], Mason R. The physiopathological basis for Zenker's diverticulum. Der Chirurg; Zeitschrift fur alle Gebiete der operativen Medizen 1999; 70: 741-746

[7] van Overbeek JJM. Meditation on the pathogenesis of hypopharyngeal (Zenker's) diverticulum and a report of endoscopic treatment in 545 patients. Ann Otol Rhinol Laryngol 1994; 103: 178-185

[8] Ferreira L, Simmons D, Baron TH. Zenker's diverticula: pathophysiology, clinical presentation, and flexible endoscopic management. Dis Esophagus 2008; 21: 1-8

[9] Smith SR, Genden EM, Urken ML. Endoscopic stapling technique for the treatment of zenker diverticulum vs standard open-neck technique: A direct comparison and charge analysis. Arch Otolaryngol Head Neck Surg 2002; 128: 141-144
[10] Costamagna G, lacopini F, Bizzotto A et al. Prognostic variables for the clinical success of flexible endoscopic septotomy of Zenker's diverticulum. Gastrointest Endosc 2016; 83: 765-773

[11] Huberty V, El Bacha S, Blero D et al. Endoscopic treatment for Zenker's diverticulum: long-term results (with video). Gastrointest Endosc 2013; 77: 701-707

[12] Halland M, Grooteman K, Baron T. Flexible endosopic management of Zenker's diverticulum: characteristics and outcomes of 52 cases at a tertiary referral center. Dis Esophagus 2016; 29: 273-277

[13] Antonello A, Ishaq S, Zanatta L et al. The role of flexible endotherapy for the treatment of recurrent Zenker's diverticula after surgery and endoscopic stapling. Surg Endosc 2016; 30: 2351-2357

[14] Ishaq S, Sultan H, Siau K et al. New and emerging techniques for endoscopic treatment of Zenker's diverticulum: State-of-the-art review. Dig Endosc 2018; 30: 449-460

[15] Sanders D, Murray T, Donnellan F. Endoscopic management of KillianJamieson diverticulum. VideoGIE 2019; 4: 364-365

[16] Tang S-j, Tang L, Chen E et al. Flexible endoscopic Killian-Jamieson diverticulotomy and literature review (with video). Gastrointest Endosc 2008; 68: 790-793

[17] Lee CK, Chung I-K, Park J-Y et al. Endoscopic diverticulotomy with an isolated-tip needle-knife papillotome (Iso-Tome) and a fitted overtube for the treatment of a Killian-Jamieson diverticulum. World J Gastroenterol 2008; 14: 6589

[18] Yang D, Draganov PV. Endoscopic Killian-Jamieson diverticulotomy using a scissor-type electrosurgical knife. Endoscopy 2018; 50: E175E176

[19] Li Q-L, Chen W-F, Zhang X-C et al. Submucosal tunneling endoscopic septum division: a novel technique for treating Zenker's diverticulum. Gastroenterology 2016; 151: 1071-1074

[20] Brieau B, Leblanc S, Bordacahar B et al. Submucosal tunneling endoscopic septum division for Zenker's diverticulum: a reproducible procedure for endoscopists who perform peroral endoscopic myotomy. Endoscopy 2017; 49: 613-614

[21] Yang J, Zeng X, Yuan X et al. An international study on the use of peroral endoscopic myotomy (POEM) in the management of esophageal diverticula: the first multicenter D-POEM experience. Endoscopy 2019; 51: 346-349

[22] Gutierrez OIB, Ichkhanian Y, Spadaccini M et al. Zenker's diverticulum per-oral endoscopic myotomy techniques: changing paradigms. Gastroenterology 2019; 156: 2134-2135

[23] Bahsi S, Rustemova N, Vosoughi K et al. Zenker's diverticulum peroral endoscopic myotomy using a scissors-type knife. Endoscopy 2019; 51: E231-E232

[24] Yang J, Novak S, Ujiki M et al. An international study on the use of peroral endoscopic myotomy in the management of Zenker's diverticulum. Gastrointest Endosc 2020; 91: 163-168

[25] Repici A, Spadaccini M, Belletrutti P] et al. Peroral endoscopic septotomy for short-septum Zenker's diverticulum. Endoscopy 2020; 52: 563-568 